\title{
Peripheral Inflammatory Yellow Exudative Retinal Coats-Like Vitreoretinopathy Misdiagnosed as Acute Retinal Necrosis in a Retinitis Pigmentosa Patient after Cataract Surgery
}

\author{
Lara Tran $^{a}$ Ioannis Papasavvas ${ }^{a} \quad J$ Johannes Fleischhauer ${ }^{b, c}$ \\ Carl P. Herbort Jr. ${ }^{a}$ \\ anflammatory and Retinal Eye Diseases, Centre for Ophthalmic Specialized Care, Lausanne, \\ Switzerland; ' ${ }^{\mathrm{b} C a b i n e t}$ d'Ophtalmologie Yverdon, Yverdon, Switzerland; 'Montchoisi \\ Teaching Centre, Lausanne, Switzerland
}

\section{Keywords}

Acute retinal necrosis $\cdot$ Coats-like disease $\cdot$ Retinitis pigmentosa

\begin{abstract}
We would like to describe a case with Coats-like exudative vitreoretinopathy after cataract surgery in a patient with retinitis pigmentosa (RP) misdiagnosed as acute retinal necrosis (ARN). A patient with RP underwent cataract surgery that was complicated by macular oedema. Following sub-Tenon's injection of triamcinolone acetonide, evolution was initially favourable. However, 2 months later, after 2 sub-Tenon's injections, the patient complained again of floaters and a drop of visual acuity. Aqueous flare measured by laser flare photometry was increased and posterior segment examination showed vitreitis, posterior haemorrhages and a temporal-inferior peripheral white-yellowish area in left eye. Serology (lgGs) for varicella-zoster virus (VZV) was slightly elevated and more so for toxoplasmosis. The whole clinical context strongly evoked ARN not excluding completely ocular toxoplasmosis. Valacyclovir and clindamycin were introduced without benefit. When examining the extreme periphery of the right fellow eye, discreet yellow lesions were also detected rendering the infectious hypothesis less probable. A vitrectomy finally excluded infectious causes and the diagnosis of Coats-like exudative vitreoretinopathy in a RP patient was retained. Increased flare despite 2 sub-Tenon's injections, the presence of micro-haemorrhages, and peripheral yellow retinal necrotic areas drew our attention away from a well-known albeit rare condition of Coats-like response in RP patients, a diagnosis which has to be considered in such circumstances.
\end{abstract}


Tran et al.: Peripheral Exudation after Cataract Surgery in Retinitis Pigmentosa

\section{Introduction}

Retinitis pigmentosa (RP) is characterized by a genetic degeneration of the retina with depigmentation and atrophy and peripheral visual field deficit [1]. Vitreitis, cystoid macular oedema (CMO), epiretinal membranes and posterior polar cataract are some of the frequent complications of RP $[1,2]$. We are reporting a case presenting with an exaggerated inflammatory reaction after cataract surgery mistaken for an infectious retinitis (ARN) due to an exudative vascular proliferation (vitreoretinopathy) and the circumstances that led to this misdiagnosis.

\section{Case Report}

A 42-year-old male patient was followed for RP. He had undergone cataract surgery 9 years previously without any complication in his right eye with a best-corrected visual acuity (BCVA) of $1.0(20 / 20)$ (Snellen chart) OU after OD cataract surgery. He complained of a decrease of visual acuity to $0.4(20 / 50)$ in his left eye due to a posterior polar cataract and underwent cataract surgery in his left eye. Visual acuity increased to $0.9(20 / 22)$ following surgery but decreased again to $0.5(20 / 40) 1$ week later due to a substantial macular oedema (retinal foveal thickness $[\mathrm{RFT}]=460 \mu \mathrm{m}$ ) shown in Figure $1 \mathrm{a}$. An abnormal anterior chamber inflammation of 38.8 photons/milliseconds ( $\mathrm{ph} / \mathrm{ms}$ ) was measured by laser flare photometry and the patient complained of floaters due to a vitreitis. In addition to corticosteroid drops (dexamethasone $0.1 \%$ TID) and oral acetazolamide ( $250 \mathrm{mg}$ ), a sub-Tenon's injection of triamcinolone acetonide ( $40 \mathrm{mg}$ ) was administered to the left eye. One week later, BCVA increased to $1.0(20 / 20)$, inflammation decreased moderately to $34.9 \mathrm{ph} / \mathrm{ms}$ and RFT decreased to $354 \mu \mathrm{m}$, as shown in Figure 1b. Two weeks later, CMO had again increased and quasi doubled to $646 \mu \mathrm{m}$ as shown in Figure 1c, with a BCVA of 0.7 (20/32) and a flare of $26.2 \mathrm{ph} / \mathrm{ms}$. A second sub-Tenon's injection of triamcinolone acetonide ( $40 \mathrm{mg}$ ) was performed with no functional nor inflammatory improvement 3 weeks later $(B C V A=0.5=20 / 40 \mathrm{ft}$ ), flare $=25.5 \mathrm{ph} / \mathrm{ms}$ ). However, RFT returned to normal, not accounting for the persisting reduced visual acuity.

Dilated fundus examination showed posterior pole haemorrhages and a temporalinferior peripheral yellow necrotic area OS shown in Figure 2a, which was suspected to correspond to ARN. Valacyclovir (1,000 mg TID) was started immediately.

The work-up showed a normal serum angiotensin-converting enzyme (10.6 U/L Normal 20-70), a slightly elevated lysozyme (24.2 mg/L Normal 9.6-17.1) and a negative QuantiFERON ${ }^{\circledR}$ Tuberculosis test $(<0.1 \mathrm{IU} / \mathrm{mL})$. Serologies showed positive but nonelevated IgGs for Epstein-Barr virus (EBV) $(1: 80 \mathrm{Neg}<1: 20)$ and a slightly elevated serology (IgGs) to varicella-zoster virus (VZV) $(8.0$ Index Neg $<0.9)$. There was no exposure to cytomegalovirus (CMV) $(<1 \mathrm{U} / \mathrm{mL} \mathrm{Neg}<6$ ) nor to herpes simplex virus (HSV) $(<0.5$ Index Neg $<0.9$ ). Toxoplasma gondii serology showed substantially elevated IgGs (300 IU/mL Neg < 1.00-3.00). After receiving the laboratory tests, clindamycin (300 mg QID) was added to the treatment. The situation did not evolve for 1 month.

The patient was only seen again another 2 months later, due to the COVID-19 lockdown. At this moment BCVA was 0.3 (20/63), flare was $17.4 \mathrm{ph} / \mathrm{ms}$ and RFT was $355 \mu \mathrm{m}$. Decreased visual acuity was due to increased vitreous opacities. Upon fundus examination OS, the posterior pole haemorrhages were slightly less and the yellowish peripheral temporalinferior lesion was stable. However, when examining the fellow right eye, similar, more discreet lesions were noted in the peripheral temporal superior fundus as shown in Figure $2 \mathrm{~b}$, making the infectious hypothesis less probable. Coat's-like evolution in a RP patient was favoured as a diagnosis, maintaining however the antiviral and antibiotic treatment.

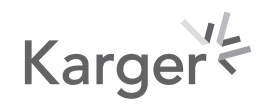




\section{Case Reports in Ophthalmology}

Tran et al.: Peripheral Exudation after Cataract Surgery in Retinitis Pigmentosa
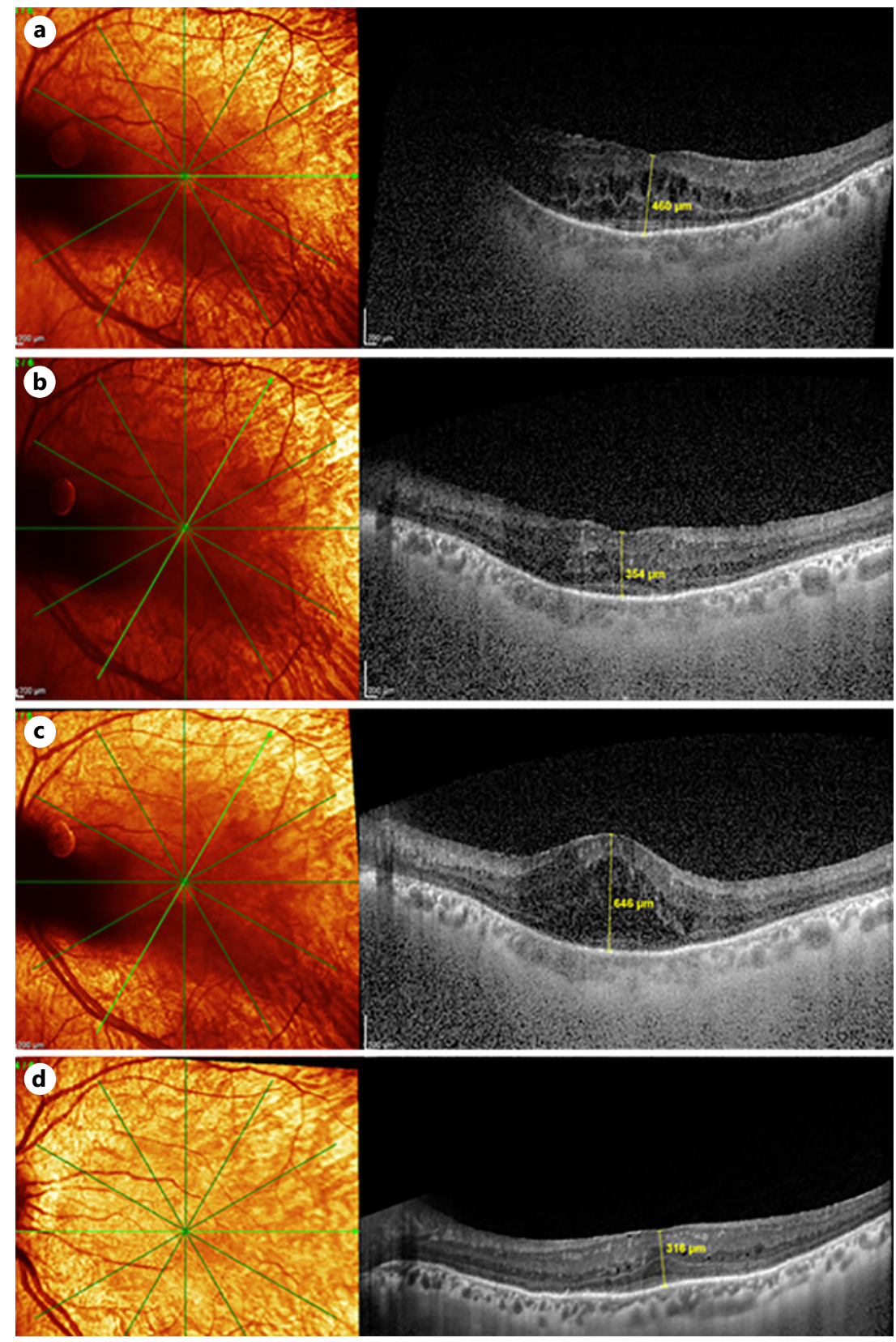

Fig. 1. Evolution of RFT during the follow-up. 10 days after surgery showing CMO (a); 1 week after sub-Tenon's triamcinolone acetonide injection (b). Decrease of CMO; 1 month after surgery showing recurrence of CMO (c); 6 months after vitrectomy (d). Further decrease of RFT. CMO, cystoid macular oedema; RFT, retinal foveal thickness.

We proposed to the patient to perform a vitrectomy on the left eye both for visual and diagnostic reasons, to confirm the hypothesis of Coats-type exudative vitreoretinopathy in RP. The patient only accepted surgery 6 weeks later. As a precaution, we maintained him under antiviral and antibiotic therapy. Pars plana vitrectomy with photocoagulation of the diseased retinal areas was performed without complication. Polymerase chain reaction analysis of the vitreous was negative for HSV1\&2, VZV, CMV, EBV, and Toxoplasma gondii. 


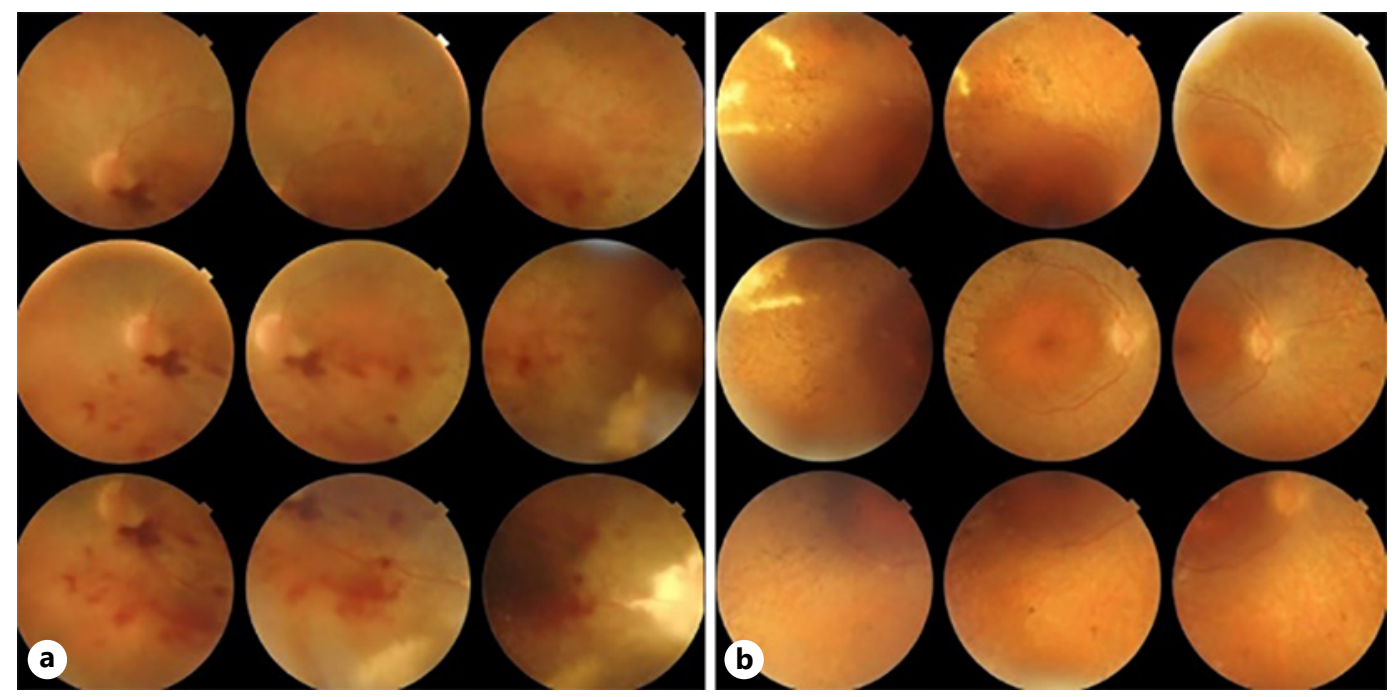

Fig. 2. a Fundus photography OS 4 weeks after surgery. Numerous haemorrhages mostly in the lower part of the retina and yellowish lesions in the inferior temporal periphery that were interpreted as ARN. b Fundus photography OD 7 weeks after surgery. Similar lesions to the lesions in the left eye that were interpreted as ARN were also found in the superior temporal area of the right eye. ARN, acute retinal necrosis.

Six months later, BCVA was 0.9 (20/22); flare remained high $(30.9 \mathrm{ph} / \mathrm{ms})$ and RFT amounted to $316 \mu \mathrm{m}$. As shown in Figure 3, the fundus lesion was replaced by a pigmented, more flat area as a result of intraoperative argon laser treatment.

\section{Discussion}

Infectious uveitis, in particular ARN, was suspected following 2 periocular corticosteroid injections for CMO in a RP patient principally because of quasi absence of response of laser flare photometry values despite the sub-Tenon's injections of triamcinolone acetonide. After 2 months of treatment with valacyclovir and clindamycin, there was no improvement and the vitreous opacity with retinal haemorrhages persisted. The discovery of similar discreet very peripheral temporal superior lesions in the fellow right eye oriented us to the rare occurrence of Coats-like exudative vasculopathy [3].

Coats disease is an idiopathic disorder characterized by leaky retinal vascular telangiectasia, aneurysms, hard exudates, and peripheral avascularity. It is typically unilateral (95-100\%) and mostly involves inferior and temporal quadrants. Young males are frequently affected and onset of symptoms appears in the first or second decades of life. It is believed that adults with Coats disease developed the disease at young age but were asymptomatic, and thus are diagnosed at an older age. Findings are similar to those in children, but the picture is less advanced. Other diseases causing exudative retinopathy should be excluded before diagnosing Coats disease in adults [4]. RP is an inherited retinal dystrophy caused by the loss of photoreceptors and characterized by retinal pigment deposit predominant in the periphery of the retina [1].

Zamorani was the first to describe the association between RP and Coats-like disease in 1956 [5]. Numerous denominations for the association have been used later including Coatslike RP [6]. Coats-like RP with telangiectatic vessels, neovascular, and exudative lesions, is an atypical form of RP and the prevalence has been documented to be $1-4 \%$ of RP patients [7, 8]. Lesions are usually found in the inferior and/or temporal quadrants in both retinae. 


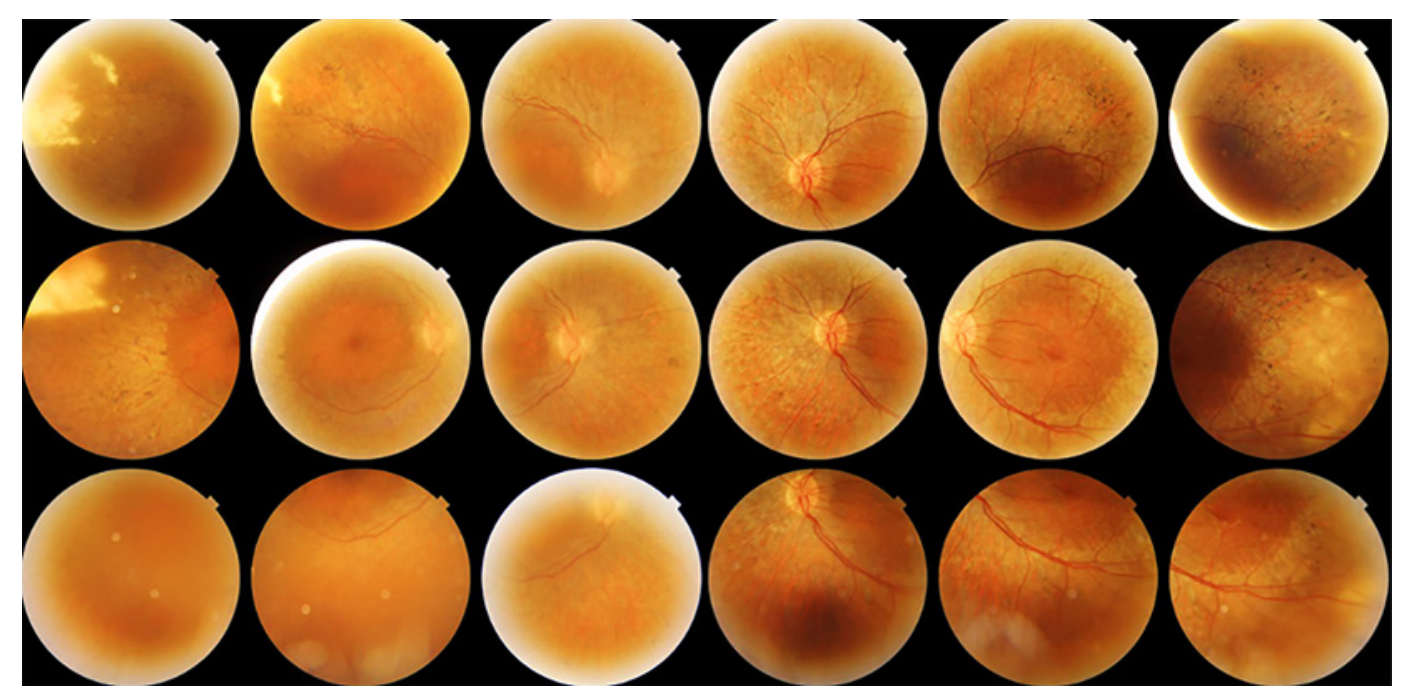

Fig. 3. Fundus photography both eyes. Left eye after pars plana vitrectomy with photocoagulation. Left panorama refers to the right eye and the left panorama to the operated eye.

Retinal haemorrhages are seen in association with vascular abnormalities that produce a Coats-like appearance. Serous retinal detachments are characterized by a striking appearance of yellow retinal or subretinal exudative deposits. However, presentation of Coats-type changes seen in RP differs from classical Coats disease: manifestations are found in older individuals without sex predilection and generally involve both eyes [6, 8-10].

Although the entity is found at a later age, a case of Coats-type secondary to RP manifesting at age 4 has been reported, preceding the diagnosis of the underlying retinal dystrophy [11]. Symptoms of Coats-like RP are varied. They most often include nyctalopia and progressive constricted visual field probably due to RP [9], but cases with mild visual difficulties on one side [10] as well as painful blind eye [12] have been described. Visual loss may be fairly rapid or may overlap a slow progressing decrease in visual acuity due to RP alone.

The cause is obscure. One hypothesis evoked is an incompetent blood-retinal barrier with subsequent leakage of serum constituents [6]. The vasodilatory response may also be caused by accumulation of toxic products from photoreceptor/RPE degeneration [8]. The second explanation is chronic microvascular leakage followed by inferior serous retinal detachment that leads to hypoxia due to retinal separation from choroid and therefore, development of telangiectatic vessel changes and further microvascular leakage [6].

We had several reasons to think about an ARN in this case, as retinal lesions occurred after local corticosteroid injection in addition to a bias due to the fact that our centre is specialized in uveitis. Studies show that ARN is characterized by a peripheral necrotizing retinitis usually due to VZV, herpes simplex virus (HSV1 or 2), and rarely CMV, or EBV. Although herpes serologies are not diagnostic, we perform them routinely as absence of exposition allows to exclude a diagnosis. We also use the serologies to detect polyclonal activation to these commensal viruses as a diagnostic element for sarcoidosis. It usually affects immunocompetent patients but is also found in individuals with altered immune function. It can be unilateral or bilateral. Symptoms include ocular pain, decreased vision, floaters, and photophobia $[13,14]$.

Extensive toxoplasmic retinitis by toxoplasmosis was also possibly suspected in presence of very high titres of IgG antibodies against Toxoplasma gondii. Extensive infection of the retina can be confused with necrotizing herpetic retinopathies. This presentation has been described most commonly in groups at risk including elderly individuals, patients with 
acquired immunodeficiency syndrome and those receiving immunosuppressive therapy but can occur in younger immunocompetent patients as well [15]. For these reasons, we decided, as a precaution to add anti-toxoplasmic antibiotics.

A recent article gave an extensive and comprehensive overview on Coats-like exudative vitreoretinopathy [9]. Our case corresponded to the characteristics described in the article as far as bilaterality and localization are concerned. However, the localization in the right fellow eye was temporal superior and the age of presentation (42 years) was at the upper limit of those reported in the article. As far as treatment was concerned laser photocoagulation was sufficiently effective in our case and anti-VEGF injections were not necessary.

\section{Conclusion}

The rare occurrence of Coats-like lesions should be kept in mind in front of a picture of an exudative vitreoretinopathy in a patient with RP. The question remaining is the role that cataract surgery had in triggering and/or amplifying the preexisting pathology of Coats-like exudative vitreoretinopathy.

\section{Acknowledgements}

Special thanks to Bruno Jeanin and Beatrice Gehrig for their help.

\section{Statement of Ethics}

The study was performed in accordance with the ethical standards laid down in the Declaration of Helsinki and in accordance with the IRB of our institution Inflammatory and Retinal Eye Diseases, Centre for Ophthalmic Specialized Care, Lausanne, Switzerland (protocol EC-COS-MTC-21-01-01, approved January 29, 2021) authorizing retrospective, anonymous and noninterventional studies. Written informed consent was obtained from the patient for publication of this case report and all the accompanying images.

\section{Conflict of Interest Statement}

The authors of this manuscript do not have any conflict of interest to declare.

\section{Funding Sources}

This manuscript did not receive any financial support.

\section{Author Contributions}

L.T. contributed in writing and collecting the data. I.P. was contributed in revision of the article. J.F. performed the surgical management and contributed to the diagnosis. C.P.H. was the main supervisor and the case report was his conception. All the authors read, edited, and approved the case report. 
Tran et al.: Peripheral Exudation after Cataract Surgery in Retinitis Pigmentosa

\section{References}

1 Hamel C. Retinitis pigmentosa. Orphanet J Rare Dis. 2006 Oct 11;1:40.

2 Liew G, Strong S, Bradley P, Severn P, Moore AT, Webster AR, et al. Prevalence of cystoid macular oedema, epiretinal membrane and cataract in retinitis pigmentosa. Br J Ophthalmol. 2019 Aug;103(8):1163-6.

3 Demirci FY, Rigatti BW, Mah TS, Gorin MB. A novel RPGR exon ORF15 mutation in a family with X-linked retinitis pigmentosa and Coats'-like exudative vasculopathy. Am J Ophthalmol. 2006 Jan 1;141(1):208-10.

4 Sen M, Shields CL, Honavar SG, Shields JA. Coats disease: an overview of classification, management and outcomes. Indian J Ophthalmol. 2019 Jun;67(6):763-71.

5 Zamorani G. Una rara associazone di retinite di coats con retinite pigmentosa. Gior Ital Oftalmol. 1956;9:429_ 43.

6 Khan JA, Ide CH, Strickland MP. Coats'-type retinitis pigmentosa. Surv Ophthalmol. 1988 Apr;32(5):317-32.

7 Kajiwara Y. Ocular complications of retinitis pigmentosa. Association with Coat's syndrome. Jpn J Clin Ophthalmol. 1980;34:947-55.

8 Pruett RC. Retinitis pigmentosa: clinical observations and correlations. Trans Am Ophthalmol Soc. 1983;81: 693-735.

9 Moinuddin O, Sathrasala S, Jayasundera KT, Branham KH, Chang EY, Qian CX, et al. Coats-like exudative vitreoretinopathy in retinitis pigmentosa: ocular manifestations and treatment outcomes. Ophthalmol Retina. 2021 Jan;5(1):86-96.

10 Banks Anderson W, Wadsworth JA, Landers MB. Retinitis pigmentosa and a retinal vasculopathy of the coats type. Adv Exp Med Biol. 1977;77:37-42.

11 Kim RY, Kearney JJ. Coats-type retinitis pigmentosa in a 4-year-old child. Am J Ophthalmol. 1997 Dec;124(6): 846-8.

12 Fogle JA, Welch RB, Green WR. Retinitis pigmentosa and exudative vasculopathy. Arch Ophthalmol. 1978 Apr 1;96(4):696-702.

13 Guex-Crosier Y, Rochat C, Herbort CP. Necrotizing herpetic retinopathies. A spectrum of herpes virus-induced diseases determined by the immune state of the host. Ocul Immunol Inflamm. 1997 Dec;5(4):259-65.

14 Bergstrom R, Tripathy K. Acute retinal necrosis. In: StatPearls [Internet]. Treasure Island, FL: StatPearls Publishing; 2020 [cited 2021 Jan 2]. Available from: http://www.ncbi.nlm.nih.gov/books/NBK470588/.

15 Holland GN. Ocular toxoplasmosis: a global reassessment. Part II: disease manifestations and management. Am J Ophthalmol. 2004 Jan;137(1):1-17. 